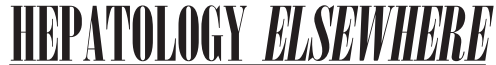

\section{Controlling Autophagy: A New Concept for Clearing Liver Disease}

Hidvegi T, Ewing M, Hale P, Dippold C, Beckett C, $\operatorname{Kemp} C$, et al. An autophagy-enhancing drug promotes degradation of mutant alpha1-antitrypsin $\mathrm{Z}$ and reduces hepatic fibrosis. Science 2010;329:229-232. (Reprinted with permission.)

\begin{abstract}
In the classical form of alpha1-antitrypsin (AT) deficiency, a point mutation in AT alters the folding of a liver-derived secretory glycoprotein and renders it aggregation-prone. In addition to decreased serum concentrations of AT, the disorder is characterized by accumulation of the mutant alpha1-antitrypsin $\mathrm{Z}$ (ATZ) variant inside cells, causing hepatic fibrosis and/or carcinogenesis by a gain-of-toxic function mechanism. The proteasomal and autophagic pathways are known to mediate degradation of ATZ. Here we show that the autophagy-enhancing drug carbamazepine (CBZ) decreased the hepatic load of ATZ and hepatic fibrosis in a mouse model of AT deficiency-associated liver disease. These results provide a basis for testing CBZ, which has an extensive clinical safety profile, in patients with AT deficiency and also provide a proof of principle for therapeutic use of autophagy enhancers.
\end{abstract}

\section{Comment}

With an expected prevalence of $0.02 \%$, alpha-1antitrypsin (AAT) deficiency is one of the most common genetic origins of liver disease in childhood and an important hereditary cause of cirrhosis and hepatocellular carcinoma in adulthood. AAT is an important serine protease inhibitor that is synthesized in the liver (normally as the protease inhibitor M [PiMM] protein), is found in the circulation at substantial levels $(>0.8 \mathrm{~g} / \mathrm{L}$ in serum), and inhibits proteolytic enzymes such as elastase released by neutrophils and macrophages (Fig. 1A). The most common initial clinical presentation of AAT deficiency is chronic obstructive pulmonary disease with typically severe, early-onset panacinar emphysema with a basilar predominance in adults. Emphysema in patients with AAT deficiency is thought to result from increased activity of neutrophil elastase in the lungs, which destroys alveolar septa and other components of the lung interstitium because of the lack of sufficient elastase inhibition by circulating AAT (Fig. 1B). ${ }^{1}$ The classic form of AAT deficiency is
EDITORS

Kris Kowdley, Seattle, WA

Geoffrey McCaughan, Newtown, Australia

Christian Trautwein, Aachen, Germany caused by a glutamate-to-lysine exchange at position 342 in the serpin peptidase inhibitor A1 gene [called the protease inhibitor $\mathrm{ZZ} \mathrm{(PiZZ)} \mathrm{genotype],} \mathrm{which}$ leads to hepatic synthesis of mutant alpha-1-antitrypsin Z (ATZ) proteins. These mutant ATZ proteins are prone to polymerization and form polymers between the mutated reactive center loop and the beta sheet of the next molecule within the endoplasmatic reticulum (ER) of the hepatocytes. ${ }^{2}$ The massive formation of insoluble aggregates of mutant ATZ proteins in the hepatocytic ER results in apoptosis, hepatic inflammation, and fibrosis/cirrhosis and strongly predisposes patients to hepatocellular carcinoma (Fig. 1B). ${ }^{3}$ The diagnosis of AAT deficiency is established by low serum AAT levels, which are measured for the screening of suspected patients; this is followed by genotyping (with PiZZ-specific polymerase chain reaction) and protein phenotyping (with isoelectric focusing gel) as verification tests. ${ }^{4}$ In liver histology, periodic acid-Schiff-positive, diastaseresistant globules containing ATZ protein polymers in hepatocytes are typically seen with AAT deficiency. ${ }^{3}$

Therapeutic options for AAT deficiency are limited at present. Patients with pulmonary manifestations are treated with standard chronic obstructive pulmonary disease drugs. In addition, augmentation therapy with regular intravenous administrations of partially purified plasma preparations highly enriched with AAT is available (Prolastin, Zemaira, and Aralast), but this therapy is expensive (ca. $\$ 60,000-\$ 150,000$ per year), and data on its effectiveness are less robust. ${ }^{1}$ Clinical trials with augmentation therapy have indicated that emphysema progression might be moderately reduced, ${ }^{5,6}$ but large studies with mortality as an endpoint are lacking at present. There is currently no therapeutic medical option available for treating liver diseases associated with AAT deficiency. Ultimately, liver transplantation is a causative therapy for AAT deficiency because it reverts the peripheral AAT deficiency and hepatic disease manifestation. Graft and patient survival rates after liver transplantation due to AAT deficiency are similar to those for other etiologies of cirrhosis. ${ }^{7}$

Several new therapeutic strategies for AAT deficiency have been proposed and investigated in the past. For instance, intravenous augmentation therapy might be replaced by intranasal drug formulations in the future, 
Fig. 1. Pathogenesis of AAT deficiency and novel therapeutic strategies for AAT deficiency-related liver disease. (A) AAT is a serine protease inhibitor. In healthy individuals, AAT is synthesized in the liver, is released into the bloodstream, inhibits various proteases (e.g., neutrophil elastase), and thereby protects the lung interstitium from elastase degradation. (B) In patients with AAT deficiency, mutant ATZ proteins polymerize in the ER of hepatocytes and cannot be released as functional proteins into the circulation; this results in an imbalance between elastases and elastase inhibition. This loss of AAT function promotes early-onset pulmonary emphysema. In the liver, polymerized ATZ proteins represent severe stress to hepatocytes (gain of function); this promotes liver injury and eventually leads to liver fibrosis, cirrhosis, or hepatocellular carcinoma. (C) Therapeutic intervention with the well-known anticonvulsant carbamazepine promotes proteolytic elimination of misfolded ATZ proteins by activating the autophagosomal degradation pathway and, to a lesser extent, the proteasomal degradation pathway in vitro and in a mouse model of AAT deficiency.
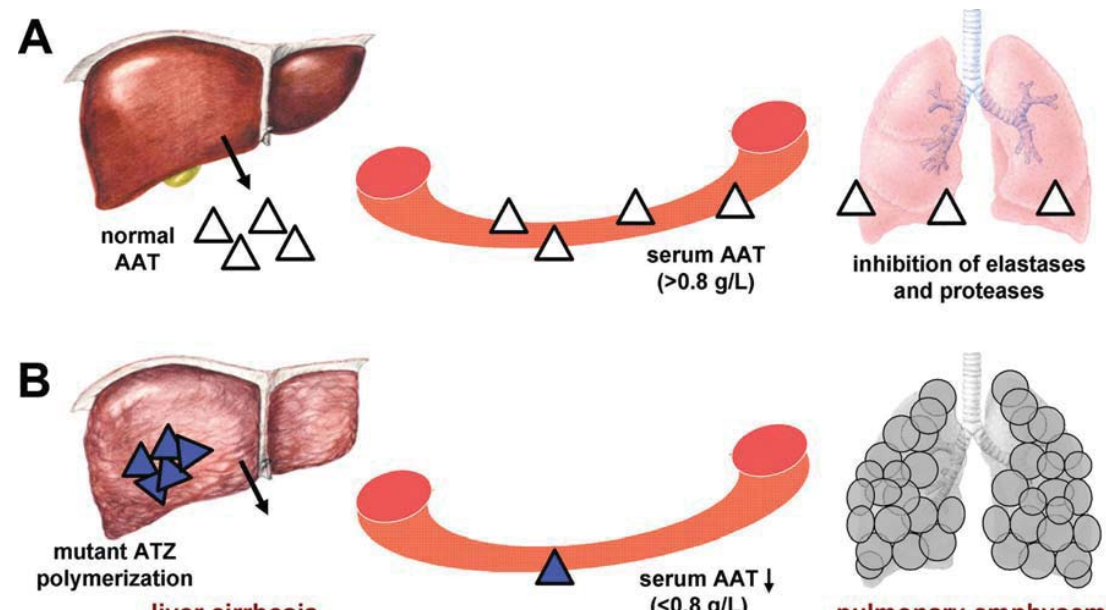

liver cirrhosis

$(<0.8 \mathrm{~g} / \mathrm{L})$

pulmonary emphysema

C

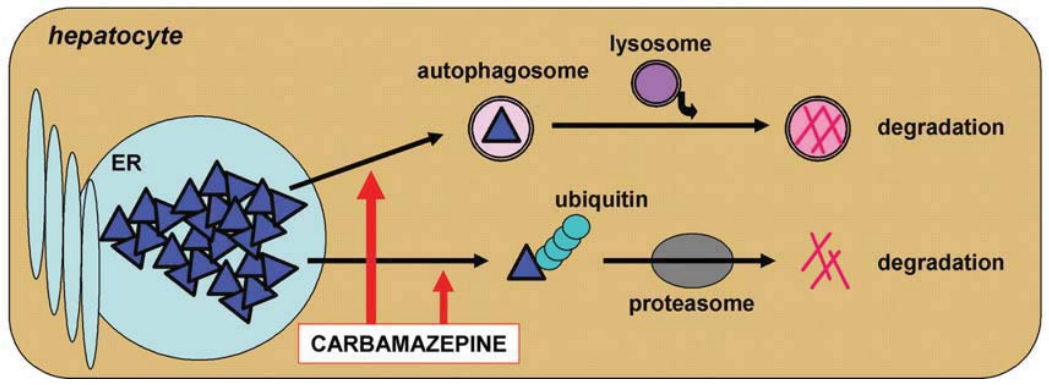

and in experimental settings, protective AAT serum levels may also be reached with gene therapy approaches (e.g., viral gene transfer into muscle cells). ${ }^{1,8}$ Targeting AAT deficiency-related liver disease has turned out to be more complex. Efficient inhibition of mutant protein polymerization is feasible in vitro but is difficult to translate into nontoxic, liver-specific drugs. ${ }^{9}$ An initial clinical trial with phenylbutyric acid as a chemical chaperone that enhanced AAT secretion in cell culture and mouse models failed because of a lack of efficacy and severe side effects in patients. ${ }^{10}$ David Perlmutter's group investigated an alternative strategy: enhancing the cellular pathways responsible for the degradation of these aberrant molecules (Fig. 1C). ${ }^{11}$ A major endogenous pathway for clearing mutant AAT polymers in the ER of hepatocytes is autophagy, a process by which the cell eradicates its own organelles through lysosomal compartments. ${ }^{12}$ The autophagosomal pathway should theoretically eliminate only misfolded monomers/polymers without affecting normal protein synthesis or secretion. Carbamazepine, a well-established anticonvulsant and mood stabilizer with an extensive clinical safety profile, is known to enhance autophagy. Perlmutter's group demonstrated that carbamazepine accelerated ATZ degradation (but not the fate of the normal AAT protein) in transfected human cells expressing AAT or ATZ and in transgenic mouse lines. ${ }^{11}$ Mechanistically, carbamazepine appeared to stimulate primarily autophagy and to a lesser extent proteasomal protein degradation $^{11}$ (Fig. 1C).

As an important step toward clinical applications, Perlmutter's group explored the effects of carbamazepine in PiZ mutant mice, a transgenic mouse model expressing human ATZ that recapitulates human AAT deficiency-related liver disease. Male PiZ mice at 5 months of age were treated with high doses of carbamazepine for 14 days. This regimen decreased the levels of ATZ proteins in the liver and typical intrahepatocytic ATZ-containing globules and increased the levels of hepatic autophagosomes. More importantly, liver fibrosis was significantly reduced in carbamazepine-treated PiZ animals. ${ }^{11}$ Interestingly, similar effects on the hepatic ATZ protein load and liver fibrosis in PiZ mice were recently reported with rapamycin, another well-established immunosuppressant drug that increases autophagy. ${ }^{13}$ Perlmutter's group was not able to reproduce a beneficial effect of rapamycin on liver fibrosis in PiZ mice, but they used a different dosing schedule for rapamycin. ${ }^{11}$

The beauty of both studies is certainly their use of well-known compounds that have already been widely tested and approved for other disorders. Unlike many experimental approaches proposed for AAT deficiency in the past, the enhancement of cellular degradation 
pathways for mutant ATZ proteins may, therefore, represent a realistic option in the near future. Nevertheless, several open questions remain. First, which of the potential drugs (carbamazepine, rapamycin, and possibly others) is most effective and best tolerated in patients with AAT deficiency? Second, is enhancing autophagy also an efficient option for advanced liver diseases (i.e., cirrhosis) in these patients? Third, how do the doses used in mice translate into humans? The carbamazepine doses necessary for beneficial effects in mice were approximately 10- to 20-fold higher (per body weight) than the therapeutic doses used in humans treated with carbamazepine for epilepsy. ${ }^{11}$ Fourth, will the activation of proteasomal degradation (observed in the carbamazepine-treated mice) affect normal hepatic protein synthesis as well? Nevertheless, the current studies provide a rationale for testing autophagy enhancers for AAT deficiency and possibly also other conditions characterized by misfolded or aggregation-prone proteins, such as Alzheimer's disease, amyloidosis, and other serpinopathies. On the other hand, autophagy inhibition could also evolve as a therapeutic concept for liver diseases in which its activation is associated with cellular senescence or transdifferentiation. ${ }^{14}$

Frank Tacke, M.D., Ph.D. Christian Trautwein, M.D. Department of Medicine III University Hospital Aachen Aachen, Germany

\section{References}

1. Silverman EK, Sandhaus RA. Alpha ${ }_{1}$-antitrypsin deficiency. N Engl J Med 2009;360:2749-2757.

2. Ekeowa UI, Freeke J, Miranda E, Gooptu B, Bush MF, Perez J, et al. Defining the mechanism of polymerization in the serpinopathies. Proc Natl Acad Sci U S A 2010;107:17146-17151.

3. Fairbanks KD, Tavill AS. Liver disease in alpha 1-antitrypsin deficiency: a review. Am J Gastroenterol 2008;103:2136-2141.

4. Eriksson S, Propst A, Sueger T, Teckman J. American Thoracic Society/European Respiratory Society statement: standards for the diagnosis and management of individuals with alpha-1 antitrypsin deficiency. Am J Respir Crit Care Med 2003;168:818-900.

5. Chapman KR, Stockley RA, Dawkins C, Wilkes MM, Navickis RJ. Augmentation therapy for alpha1 antitrypsin deficiency: a meta-analysis. COPD 2009;6:177-184.

6. Dirksen A, Piitulainen E, Parr DG, Deng C, Wencker M, Shaker SB, et al. Exploring the role of CT densitometry: a randomised study of augmentation therapy in alpha1-antitrypsin deficiency. Eur Respir J 2009; 33:1345-1353.

7. Kemmer N, Kaiser T, Zacharias V, Neff GW. Alpha-1-antitrypsin deficiency: outcomes after liver transplantation. Transplant Proc 2008;40: 1492-1494

8. Gooptu B, Lomas DA. Conformational pathology of the serpins: themes, variations, and therapeutic strategies. Annu Rev Biochem 2009; 78:147-176.
9. Mallya M, Phillips RL, Saldanha SA, Gooptu B, Brown SC, Termine DJ, et al. Small molecules block the polymerization of $\mathrm{Z}$ alpha1-antitrypsin and increase the clearance of intracellular aggregates. J Med Chem 2007;50:5357-5363.

10. Teckman JH. Lack of effect of oral 4-phenylbutyrate on serum alpha1-antitrypsin in patients with alpha-1-antitrypsin deficiency: a preliminary study. J Pediatr Gastroenterol Nutr 2004;39:34-37.

11. Hidvegi T, Ewing M, Hale P, Dippold C, Beckett C, Kemp C, et al. An autophagy-enhancing drug promotes degradation of mutant alpha 1-antitrypsin Z and reduces hepatic fibrosis. Science 2010;329:229-232.

12. Sifers RN. Clearing conformational disease. Science 2010;329:154-155.

13. Kaushal S, Annamali M, Blomenkamp K, Rudnick D, Halloran D, Brunt EM, et al. Rapamycin reduces intrahepatic alpha-1-antitrypsin mutant $\mathrm{Z}$ protein polymers and liver injury in a mouse model. Exp Biol Med (Maywood) 2010;235:700-709.

14. Sasaki M, Miyakoshi M, Sato Y, Nakanuma Y. Autophagy mediates the process of cellular senescence characterizing bile duct damages in primary biliary cirrhosis. Lab Invest 2010;90:835-843.

Copyright (ㄷ 2011 by the American Association for the Study of Liver Diseases. View this article online at wileyonlinelibrary.com

DOI 10.1002/hep.24090

Potential conflict of interest: Nothing to report.

\section{Identifying Who Is at Risk of Drug-Induced Liver Injury: Is Human Leukocyte Antigen Specificity the Key?}

Singer JB, Lewitzky S, Leroy E, Yang F, Zhao X, Klickstein $L$, et al. A genome-wide study identifies HLA alleles associated with lumiracoxib-related liver injury. Nature 2010;42:711-714. Available at: www. nature.com (Reprinted with permission.)

\section{Abstract}

Lumiracoxib is a selective cyclooxygenase- 2 inhibitor developed for the symptomatic treatment of osteoarthritis and acute pain. Concerns over hepatotoxicity have contributed to the withdrawal or nonapproval of lumiracoxib in most major drug markets worldwide. We performed a case-control genome-wide association study on $\mathbf{4 1}$ lumiracoxib-treated patients with liver injury (cases) and 176 matched lumiracoxib-treated patients without liver injury (controls). Several SNPs from the MHC class II region showed strong evidence of association (the top SNP was rs9270986 with $P=2.8 \times 10^{-10}$. These findings were replicated in an independent set of 98 lumiracoxib-treated cases and 405 matched lumiracoxib-treated controls (top SNP rs3129900, $P=4.4 \times 10^{-12}$. Fine mapping identified a strong association to a common HLA haplotype (HLA-DRB1*1501HLA-DQB1*0602-HLA-DRB5*0101-HLA-DQA1*0102, most significant allele $P=6.8 \times 10^{-25}$, allelic odds ratio $=5.0$, 95\% CI 3.6-7.0). These results offer the potential to improve the safety profile of lumiracoxib by identifying individuals at elevated risk for liver injury and excluding them from lumiracoxib treatment.

\section{Comment}

Despite its relatively infrequent occurrence, druginduced liver injury (DILI) is the leading cause of 
acute liver injury in the United States, an important cause of sporadic acute hepatitis in the community, a source of diagnostic and therapeutic challenges for treating clinicians and a common reason for premarketing and postmarketing drug withdrawals for pharmaceutical companies.

The selective cyclooxygenase-2 (COX-2) inhibitor, lumiracoxib, joins the long list of nonsteroidal antiinflammatory drugs (NSAIDs) (benoxaprofen, bromfenac, ibufenac) withdrawn due to their association with DILI. ${ }^{1}$ When first introduced, lumiracoxib appeared to fulfill many of the desired attributes of an NSAID: three-fold to four-fold lower gastrointestinal (ulcerrelated) complications than naproxen and ibuprofen and a slightly better cardiovascular disease track record than rofecoxib, another recently withdrawn COX-2 inhibitor. ${ }^{2}$ Ironically, although gastroenterologists should have welcomed the introduction of such an agent, it turns out that lumiracoxib has the potential for rare but serious hepatotoxicity. Worldwide, at least 20 cases of severe DILI associated with lumiracoxib have been reported, including 14 with acute liver failure, two deaths, and three liver transplants. ${ }^{3}$ Most cases occurred several months after starting lumiracoxib, but early presentations were also noted. Many cases involved daily doses exceeding $100 \mathrm{mg}$, but severe DILI was also reported in those patients who were prescribed $100 \mathrm{mg} /$ day.

The U.S. Food and Drug Administration (FDA) issued a "nonapprovable" letter for lumiracoxib in 2007. Although the passing of one more NSAID is likely to be soon forgotten, there are two lessons to be learned for prescribers. Yet again, postmarketing surveillance has identified serious instances of DILI that were not foreseen in clinical trials. In the large TARGET (Therapeutic Arthritis Research and Gastrointestinal Event Trial) study, 2.6\% had aminotransferase (AT) elevations greater than three times the upper limit of normal $(3 \times \mathrm{ULN})$. There were six cases of probable or possible "clinical hepatitis", but all resolved with cessation of the drug, and there were no reports of liver failure. Parallels can be drawn with troglitazone. ${ }^{4}$ However, whereas the relative rarity and unpredictability of many or now most causes of DILI has been recognized for more than 50 years, the genetic basis for such a host of susceptibility factors has been slow to document reliably since rare family clustering studies and indirect susceptibility tests were reported at least 25 years ago. ${ }^{5}$ The addition of lumiracoxib to the growing list of agents for which susceptibility to DILI has been linked to human leukocyte antigen (HLA) genotypes, as reviewed recently in HEPATOLOGY, ${ }^{6}$ provokes further consideration of the mechanistic significance and clinical utility of such associations.

The observations of Singer et al., who carried out a pharmacogenetic case-control analysis of participants enrolled into the two TARGET trials, are of particular interest. $^{7}$ In the first phase of their study, 41 subjects with serum alanine aminotransferase (ALT) or aspartate aminotransferase (AST) $>5 \times$ ULN ("cases") and 176 age-matched, sex-matched, race-matched, and clinical trial-matched individuals (who took lumiracoxib but had normal ALT/AST; "controls") were recruited for a genome-wide association study (GWAS). This was performed using the Affymetrix assay 6.0, which can detect more than 900,000 singlenucleotide polymorphisms (SNPs). Several SNPs from the major histocompatibility complex (MHC) class II region on chromosome 6 were significantly represented in cases with DILI, the leading contender being rs9270986 $\left(P=2.8 \times 10^{-10}\right)$. Thirteen SNPs, including seven from phase 1 of the trial, were reevaluated in the second (validation) phase of the study, involving 98 cases and 405 lumiracoxib-exposed controls, respectively. Cases were defined here by ALT/AST $>3 \times$ ULN. The results of the replication phase confirmed the association of lumiracoxib-related DILI with the principal SNPs identified earlier, but did not find a similar relationship with cases of DILI drawn from small groups of controls receiving ibuprofen $(n=18)$ or naproxen $(n=9)$. Finally, fine mapping of the top SNPs showed strong association with a well-characterized MHC haplotype (HLA-DRB1*1501-HLADQB ${ }^{*} 0602$-HLA-DRB5 ${ }^{*} 0101$-HLA-DQA ${ }^{*} 0102$; most significant allele $P=6.8 \times 10^{-25}$, allelic odds ratio $=5.0 ; 95 \%$ confidence interval $[\mathrm{CI}]=3 \cdot 6-7.0)$. Of these alleles, HLA-DQA1*0102 had the best negative predictive value (99\%) and sensitivity $(73.6 \%)$ in identifying cases at risk.

Before examining the implications of this study, it is worthwhile to look at the wider perspective of host/drug factors influencing susceptibility to DILI. Although the total dose of drug is critical in dosedependent hepatotoxicity (e.g., acetaminophen), the relevance of this to idiosyncratic drug reactions is overshadowed by other host characteristics such as age, sex, comorbid illnesses, and coprescribed medications. ${ }^{8} \mathrm{~A}$ genetic predisposition to DILI is well recognized for drugs (phenytoin, sulfonamides) linked to hepatic injury as part of systemic hypersensitivity ("reactive metabolite syndrome") and has been recognized for halothane. ${ }^{5}$ Other than these examples, the genetic contribution to DILI has only slowly been recognized, perhaps partly because of studies in the 1990s that 
Table 1. HLA Alleles Associated With DILI

\begin{tabular}{llll}
\hline Drug & \multicolumn{1}{c}{ Therapeutic Group } & HLA Allele & Odds Ratio for Developing DILI (95\% Cl) \\
\hline Ticlopidine & Antiplatelet agent & HLA A*3303 & $36.5(7.3-184)$ \\
Flucloxacillin* & Antibiotic & HLA B*5701 & $80.6(22.8-284.9)$ \\
Amoxicillin-clavulanate & Antibiotic & HLA-DRB1*15 & $2.3(1.0-5.26)$ \\
Lumiracoxib* & NSAID & HLA-DQA1*0102 & $6.3(4.1-9.6)$ \\
Ximelagatran* & Oral direct thrombin inhibitor & HLA-DRB1*0701 & 4.4 \\
Lapatinib* & Tyrosine kinase inhibitor used & HLA-DQA1*201 & $9(3.2-27.4)$ \\
& in advanced breast cancer & & \\
\hline
\end{tabular}

*Genome-wide association study.

showed a lack of association between HLA markers and DILI. ${ }^{9}$ Although some HLA markers were overrepresented in some cases (e.g., HLA A-11 in 75\% of cases of diclofenac hepatitis), no overall association between specific HLA alleles and DILI could be discerned. Another limitation was the use of insensitive serological methods to determine HLA status instead of high-resolution genotyping on large case and control populations that is currently favored. These studies were also underpowered to detect meaningful associations with individual drugs. This poses a considerable challenge because cases of DILI are infrequent (typically between 1 and 10 per 100,000 persons exposed) and collating a case series requires considerable collaborative efforts. Furthermore, careful case definition is necessary; for DILI, this itself poses a considerable challenge.

Studies have usually used one of the causality scoring systems, such as the CIOMS (Council for International Organizations of Medical Sciences), which although laudable in many respects, lack sensitivity and specificity for several phenotypes of DILI, as reviewed elsewhere. ${ }^{10}$ Thus, in the lumiracoxib study, only 41 cases were included, and these were defined by ALT/AST changes and not by "clinical hepatitis", which differs from another GWAS study that enrolled patients with flucloxacillin-associated DILI who had clinical features of liver disease. ${ }^{11}$ As a result of these logistic limitations, it is pertinent to consider whether the reported association between lumiracoxib-related AT elevations (DILI) and the HLA allele/extended haplotype is clinically meaningful. This cannot be conclusively determined from a study of this size, but supportive arguments have been put forward. Singer et al. noted the increasing sensitivity with increases in ALT rise; all patients with ALT $>20 \times$ ULN carried the specific HLA haplotype. Also, all three cases with substantial serum bilirubin increases that fulfilled "Hy's law" (ALT/ AST $>3 \times$ ULN; serum bilirubin $>2$ ULN), a reliable marker for high probability of significant hepatotoxicity, ${ }^{12}$ also carried the implicated HLA alleles. In other respects, the study by Singer and colleagues fulfills the necessary requisites for a GWAS: proper case definition (albeit by biochemical and not clinical presentation), matched controls in a ratio of cases:controls of 1:4, use of a replication cohort, and correction of $P$ value for multiple comparisons. ${ }^{13}$

At the end of all this, what are the implications of this study in terms of pathogenesis of DILI and whether these observations can be used to prevent DILI in the future?

The physiological role of HLA class I (A, B, and C) and class II (DP, DQ, and DR) molecules on the cell surface is to present endogenous (class I) or exogenous material such as drugs (class II) to $\mathrm{T}$ lymphocytes through engagement with the $\mathrm{T}$ cell receptor. Recognition of small molecular weight drug/drug metabolites by $\mathrm{T}$ cells will occur either if presented in combination with a protein ("hapten" hypothesis) and MHC class II molecule (MHC peptide-complex), or by direct engagement with the MHC molecule ("pharmaceutical interaction" concept). ${ }^{14}$ In either scenario, it is conceivable that alterations in $\mathrm{MHC}$ alleles will disrupt proper drug- $\mathrm{T}$ cell engagement. The species differences in MHC restriction would account for the failure to predict human hepatotoxicity despite apparent safety in animal models.

In the study by Singer et al., there were no functional analyses that could shed light on the precise mechanisms of lumiracoxib-related DILI. It is, however, interesting that lumiracoxib is bioactivated to a reactive quinone imine, ${ }^{15}$ and possibly noteworthy that the structure of lumiracoxib closely resembles diclofenac. The latter is also associated with hepatotoxicity, and has metabolic pathways that can generate reactive metabolites capable of forming adducts with hepatic proteins and evoking an immune response. ${ }^{16}$ On the other hand, lumiracoxib shows no structural similarity to abacavir, which is associated with a severe cutaneous hypersensitivity reaction linked to one of the same HLA haplotypes (HLA-B*5701) as lumiracoxib. Interestingly, association with HLA-B*5701 is also shared with flucloxacillin, a synthetic penicillin associated with severe cases of drug-induced cholestasis. ${ }^{11}$ 
Unlike autoimmune hepatitis where specific HLA alleles can determine disease severity or treatment outcome, only limited genotype-phenotype correlations have been noted for instances of DILI. Interestingly, one of the same HLA haplotypes associated with lumiracoxib toxicity (HLA-DRB1*1501) is overrepresented among cases of liver injury resulting from amoxicillinclavulanate. ${ }^{17}$ However, the latter causes early onset ( $<25$ days) liver toxicity and has a completely different histologic pattern (mainly cholestatic injury), which differs from the usual late-onset hepatocellular reaction with lumiracoxib. Other recent associations of specific HLA alleles with DILI are listed in Table 1 and have been reviewed recently in Hepatology. ${ }^{6}$ It should be pointed out that not all HLA phenotypes are associated with increased susceptibility to DILI; HLADRB ${ }^{*} 07$ family of alleles conferred a reduced risk of DILI with amoxicillin-clavulanate as compared with population controls and treated nonaffected cases (odds ratio $=0.26$ and 0.18 , respectively). ${ }^{18}$ Overall, in most cases of DILI, the presence of a particular HLA allele is neither sufficient nor necessary for a particular adverse effect to occur. In addition to known and unknown host and environmental factors, the contributions of polymorphisms within drug-metabolizing systems, biliary transporters, and both innate and adaptive immune response pathways, as well as antioxidant, antiapoptosis, and other cell protective genes, need to be considered. ${ }^{6}$ It also remains possible that particular HLA alleles are in linkage disequilibrium with cardinal "susceptibility genes", as turned out to be the explanation for the association between HLA A3 and C282Y, which led to the common form of genetic hemochromatosis. ${ }^{19}$

Many consider the era of pharmacogenomic explanations for idiosyncratic adverse drug reactions to have begun with recognition of the association between hypersensitivity reactions to abacavir, a human immunodeficiency virus (HIV) protease inhibitor and HLA B $5701 .{ }^{20}$ Screening subjects for this HLA allele and withholding abacavir from those carrying it has almost completely abolished such reactions. However, unlike most cases of DILI, abacavir reactions are quite frequent $(5 \%)$, and use of common agents like antimicrobials and NSAIDs is not usually subject to the same complex considerations as highly active antiretroviral therapy for HIV. A similar HLA-based screening strategy to exclude DILI is therefore unlikely to be logistically plausible or cost-effective unless screening costs become cheaper. In the case of lumiracoxib, excluding carriers of the HLA-DQA ${ }^{*} 0102$ allele would reduce the frequency of DILI to $1 \%$ but at the expense of excluding a considerable proportion (34\%) of carriers, because less than $6 \%$ would actually develop hepatotoxicity. An alternative pharmacogenetic strategy is to restrict testing to those at increased risk of adverse drug reactions. For example, the FDA recommends screening Han Chinese patients for HLAB*1502 before starting carbamazepine. ${ }^{21}$ Such screening is likely to be cost-effective because the allele in question is relatively common in that ethnic group (8\%$12 \%)$ and, further, the odds ratio of developing a severe cutaneous reaction in persons carrying that allele is extremely high $(>2500)$. This strategy would be useless in Caucasians who do not carry that specific HLA allele but also can develop similar reactions with carbamazepine. Likewise, a selective screening protocol cannot be applicable to lumiracoxib recipients because of the failure to identify specific characteristics that could be associated with a risk of DILI.

In the final analysis, routine pharmacogenetic testing would come down to costs, availability of alternative treatment options, and logistics (turn around times). Promising times lie ahead for the prospects of pharmacogenomic discovery to help unravel the multiple interactive mechanisms of DILI, ${ }^{6}$ but their impact on preventing DILI in the near future is still likely to be limited.

\author{
Shivakumar ChitTuri, M.D., FRACP \\ Geoffrey C. Farrell, M.D., FRACP \\ Gastroenterology and Hepatology Unit \\ The Canberra Hospital and the \\ Australian National University Medical School \\ Canberra, ACT, Australia
}

\section{References}

1. Teoh NC, Farrell GC. Hepatotoxicity associated with non-steroidal anti-inflammatory drugs. Clin Liver Dis 2003;7:401-413.

2. Schnitzer TJ, Burmester GR, Mysler E, Hochberg MC, Doherty M, Ehrsam E, et al.Comparison of lumiracoxib with naproxen and ibuprofen in the Therapeutic Arthritis Research and Gastrointestinal Event Trial (TARGET), reduction in ulcer complications: randomised controlled trial. Lancet 2004;364:665-674.

3. Drug safety update: Lumiracoxib hepatotoxicity. Available from http:// www.mhra.gov.uk/home/idcplg?IdcService=GET_FILE\&dDocName= CON2033077\&RevisionSelectionMethod=LatestReleased. Accessed December 2, 2010.

4. Graham DJ, Green L, Senior JR, Nourjah P. Troglitazone-induced liver failure: a case study. Am J Med 2003;114:299-306.

5. Farrell GC, Prendergast D, Murray M. Halothane hepatitis. Detection of a constitutional susceptibility factor. N Engl J Med 1985;313: 1300-1314.

6. Russmann S, Jetter A, Kullak-Ublick GA. Pharmacogenetics of druginduced liver injury. Hepatology 2010;52:748-761.

7. Singer JB, Lewitzky S, Leroy E, Yang F, Zhao X, Klickstein L, et al.A genome-wide study identifies HLA alleles associated with lumiracoxibrelated liver injury. Nature 2010;42:711-714.

8. Chalasani N, Björnsson E. Risk factors for idiosyncratic drug-induced liver injury. Gastroenterology 2010;138:2246-2259. 
9. Berson A, Fréneaux E, Larrey D, Lepage V, Douay C, Mallet C, et al.Possible role of HLA in hepatotoxicity. An exploratory study in 71 patients with drug-induced idiosyncratic hepatitis. J Hepatol 1994;20: 336-342.

10. Chitturi S, Farrell GC. Drug induced liver disease. In: Schiff ER, Sorrell MF, Maddrey WC, eds. Schiff's Diseases of the Liver, ed. 10. Philadelphia: LippincottWilliams \& Wilkins; 2007.

11. Daly AK, Donaldson PT, Bhatnagar P, Shen Y, Pe'er I, Floratos A, et al.HLA-B*5701 genotype is a major determinant of drug-induced liver injury due to flucloxacillin. Nat Genet 2009;41:816-819.

12. Temple R. Hy's law: predicting serious hepatotoxicity. Pharmacoepidemiol Drug Saf 2006;15:241-243.

13. Pearson TA, Manolio TA. How to interpret a genome-wide association study. JAMA 2008;299:1335-1344.

14. Uetrecht J. Idiosyncratic drug reactions: past, present, and future Chem Res Toxicol 2008;21:84-92.

15. Kang P, Dalvie D, Smith E, Renner M. Bioactivation of lumiracoxib by peroxidases and human liver microsomes: identification of multiple quinone imine intermediates and GSH adducts. Chem Res Toxicol 2009;22:106-117.

16. Aithal GP, Ramsay L, Daly AK, Sonchit N, Leathart JB, Alexander G, et al. Hepatic adducts, circulating antibodies, and cytokine polymorphisms in patients with diclofenac hepatotoxicity. Hepatology 2004; 39:1430-1440.

17. Andrade RJ, Lucena MI, Alonso A, García-Cortes M, García-Ruiz E, Benitez R, et al. HLA class II genotype influences the type of liver injury in drug-induced idiosyncratic liver disease. Hepatology 2004;39:1603-1612.

18. Donaldson PT, Daly AK, Henderson J, Graham J, Pirmohamed M, Bernal W, et al.Human leucocyte antigen class II genotype in susceptibility and resistance to coamoxiclav-induced liver injury. J Hepatol 2010; 53:1049-1053.

19. Simon M, Bourel M, Fauchet R, Genetet B. Association of HLA-A3 and HLA- B14 antigens with idiopathic haemochromatosis. Gut 1976; 17:332-334.

20. Mallal S, Phillips E, Carosi G, Molina JM, Workman C, Tomazic J, et al.HLA-B*5701 screening for hypersensitivity to abacavir. N Engl J Med 2008;358:568-579.

21. Tohkin M, Ishiguro A, Kaniwa N, Saito Y, Kurose K, Hasegawa R. Prediction of severe adverse drug reactions using pharmacogenetic biomarkers. Drug Metab Pharmacokinet 2010;25:122-133.

Copyright $(\subset 2011$ by the American Association for the Study of Liver Diseases. View this article online at wileyonlinelibrary.com. DOI 10.1002/hep.24094

Potential conflict of interest: Nothing to report.

\section{The Genetics of Primary Biliary Cirrhosis: The Revolution Moves On}

Hirschfield GM, Liu X, Han Y, Gorlov IP, Lu Y, Xu C, et al. Variants at IRF5-TNPO3, 17q12-21 and MMEL1 are associated with primary biliary cirrhosis. Nat Genet 2010;42:655-657. (Reprinted with permission.)

\section{Abstract}

We genotyped individuals with primary biliary cirrhosis and unaffected controls for suggestive risk loci (genome-wide association $\left.P<1 \times 10^{-4}\right)$ identified in a previous genome-wide association study. Combined analysis of the genome-wide association and replication datasets identified IRF5-TNPO3 (combined $\left.P=8.66 \times 10^{-13}\right), 17 q 12-21$ (combined $P=3.50 \times$ $10^{-13}$ ) and MMEL1 (combined $P=3.15 \times 10^{-8}$ ) as new pri- mary biliary cirrhosis susceptibility loci. Fine-mapping studies showed that a single variant accounts for the IRF5-TNPO3 association. As these loci are implicated in other autoimmune conditions, these findings confirm genetic overlap among such diseases.

Liu X, Invernizzi P, Lu Y, Kosoy R, Lu Y, Bianchi I, et al. Genome-wide meta-analyses identify three loci associated with primary biliary cirrhosis. Nat Genet 2010;42:658-660. (Reprinted with permission.)

\section{Abstract}

A genome-wide association screen for primary biliary cirrhosis risk alleles was performed in an Italian cohort. The results from the Italian cohort replicated $I L 12 A$ and $I L 12 R B$ associations, and a combined meta-analysis using a Canadian dataset identified newly associated loci at $\operatorname{SPIB}\left(P=7.9 \times 10^{-11}\right.$, odds ratio $(\mathrm{OR})=1.46)$, IRF5-TNPO3 $\left(P=2.8 \times 10^{-10}, \mathrm{OR}\right.$ $=1.63)$ and $17 q 12-21\left(P=1.7 \times 10^{-10}, O R=1.38\right)$.

\section{Comment}

The 2009 publication of the first genome-wide association study (GWAS) of primary biliary cirrhosis (PBC) represented a key point in the evolution of our understanding of the genetic basis and thus pathogenesis of this disease. ${ }^{1}$ This landmark study identified, in a reproducible fashion, genetic associations between PBC and human leukocyte antigen as well as polymorphisms in the genes encoding the interleukin-12 (IL-12) $\alpha$-chain and the IL-12 receptor $\beta$-chain. Two recent publications from Canadian, American, and Italian groups add an important further dimension to our knowledge base with respect to the genetic basis of $\mathrm{PBC}$ and build on the original study. ${ }^{2,3}$ Taken together, these two new studies replicate the original genetic associations with the IL-12 pathway, and importantly, through individual and combined analyses, they identify further associated loci. Critically, the newly identified loci are again associated with the biology of the interaction between antigen-presenting cells (APCs) and $\mathrm{CD}^{+}{ }^{+} \mathrm{T}$ cells, which is thought to be critical to the development of the autoreactive immune responses underpinning PBC. ${ }^{4}$ The advent of these new data make now a good time to reflect on what we now know and to identify potential future directions for research. Three important observations can be made about our new understanding of the genetic associations of $\mathrm{PBC}$.

The first observation is the striking consistency between the findings of the original GWAS and those of the current Italian/American study. This sense of a single uniform association pattern for $\mathrm{PBC}$ is further reinforced by the as yet unpublished findings of a large UK GWAS, which again replicates all findings made to date. 
The strength and consistency of the findings in fully independent studies are themselves worthy of comment. This finding would confirm the view from population and twin-based studies that there is a significant genetic contribution to PBC. ${ }^{5,6}$ A further significant factor, however, in the clarity of the findings is the fact that $\mathrm{PBC}$ probably does constitute a single disease entity across different populations. Another factor is also likely to play a role in the consistency of the findings between the studies: the simplicity and accuracy of the diagnostic criteria for PBC. The combination of antimitochondrial antibodies on immunofluorescence (or anti-M2 antibodies on an enzyme-linked immunosorbent assay) and cholestatic liver function tests is $95 \%$ sensitive and specific for the diagnosis of $\mathrm{PBC}^{7}$ This degree of diagnostic accuracy, which stands in contrast to many other disease states for which GWASs have given rise to weaker and more contradictory findings ${ }^{8}$ and for which diagnosis at the level of accuracy needed to avoid confounding genetic studies is more complicated, has the important benefit of effectively excluding the false-positive assignment of disease status, which introduces error and reduces power in GWASs. One of the conclusions that can be drawn from the PBC GWASs published to date is, therefore, that this disease is in fact an extremely valuable model with which to study genetic contributions to the pathogenesis of autoimmune disease.

The second observation that can be made is related to the nature of the associations found and replicated to date, all of which are for genes encoding proteins implicated in antigen presentation by APCs and the resultant induction of $\mathrm{T}$ cell immune responses. Major histocompatibility complex is clearly critical for the presentation of peptide epitopes, whereas the IL-12 pathway plays a key role in shaping the phenotype of the resulting $\mathrm{T}$ cell response and is essential for the development of proinflammatory $\mathrm{T}$ helper 1 (Th-1) type immune responses. The novel genetic associations with interferon regulatory factor 5 (IRF5)-transportin 3, $S P I B$, and the $17 \mathrm{q} 12-21$ chromosomal region that are reported in the two new studies (individually and in a meta-analysis) continue this theme. SPIB is a transcription factor that plays a role, among many others, in the pathway for the differentiation of plasmacytoid dendritic cells, which can also mediate and modulate the expression of CD40 (its interaction with the CD40 ligand has previously been identified as a key costimulatory/effector pathway in PBC). ${ }^{9-11}$ IRF5 plays a key role in the innate immune response as part of the tolllike receptor signaling pathway ${ }^{12}$ and mediates apoptosis induced by tumor necrosis factor-related apoptosisinducing ligand. ${ }^{13}$ Strikingly, IRF5 loci have previously been shown to be associated with autoimmune disease in the form of systemic lupus erythematosus, systemic sclerosis, and Sjögren's syndrome ${ }^{14,15}$; all these conditions are known to be associated with $\mathrm{PBC}^{16}$ The 17q12-21 region contains a number of potentially biologically relevant genes and has itself previously been shown to be associated with other inflammatory and autoimmune diseases, including rheumatoid arthritis. ${ }^{17}$ What is striking is that all the identified associations are related to the immune response and, in particular, to the interactions relating to APC development, APC activation, epitope presentation, and the phenotype of the resulting $\mathrm{T}$ cell response. The inescapable conclusion is, therefore, that $\mathrm{PBC}$ is an immune disease, at least in genetic terms. It will be interesting to see whether the UK GWAS, which will be the largest to date and thus will have significantly augmented statistical power, identifies further genetic associations within this key pathway.

The third observation is related to a number of associations that can be hypothesized to be relevant to the pathogenesis of $\mathrm{PBC}$ on the basis of our ideas about its biology but that are not seen. To date, with the important caveat remaining about the power of GWASs necessary to identify all associations, no susceptibility loci related to the biology of the pyruvate dehydrogenase complex (the key disease autoantigen $^{18}$ ), the biology of biliary epithelial cells (the target cells for damage in $\mathrm{PBC}^{4}$ ), or potential disease phenotype-controlling factors (the phenotype can have a big impact on the probability of a diagnosis being made) such as biliary transporter genes have been identified. It may be that a better powered GWAS or pathway analysis could identify such factors, but until this occurs, what we will see is an exclusively immunoregulatory portfolio of genetic associations.

What, therefore, do we know and still not know about PBC after the publication of these genetic studies? What is now absolutely clear (if it was not clear before) is that PBC is likely a disease of immune dysregulation. What predisposes a person to it is variability in the genes encoding the key proteins that regulate the normal immune response to an antigen. What we tantalizingly do not know yet and will not know until the results of functional studies emerge is whether $\mathrm{PBC}$ is associated with augmented Th- 1 phenotype immunity or impairment. This is critical because it is conceptually possible to link augmented immunity and impaired immunity of the Th-1 phenotype with the pathogenesis of $\mathrm{PBC}$ through conventional autoreactivity and an impaired response to a pathogen model, respectively. This next stage of functional study is, 
therefore, absolutely critical for the future direction of $\mathrm{PBC}$ immunology research.

The value of the GWAS approach has been questioned recently, with the argument put forward that it "flatters to deceive" and fails to deliver the anticipated paradigm shift in disease understanding. ${ }^{8}$ In this sense, therefore, $\mathrm{PBC}$ represents a triumph for GWASs because the PBC studies are likely to turn out to be landmarks in our understanding of the disease. It is also likely that the findings will translate into new approaches to therapy sooner than GWAS findings typically do, with modulation of the IL-12 pathway representing one obvious potential approach. The findings of these studies also, however, suggest that PBC may be not only an important disease to study in its own right but also an important paradigm for our understanding of immune regulation in humans as a result of its homogeneity and the diagnostic accuracy. It is often forgotten that PBC was a landmark disease in the study of autoimmunity and represented one of the first disease settings in which autoantibodies were described and in which the autoantigens associated with human disease were identified. ${ }^{19-21}$ We may now be at the point at which PBC returns to the forefront of the study of the mechanistic immunobiology of autoimmunity. These are interesting times.

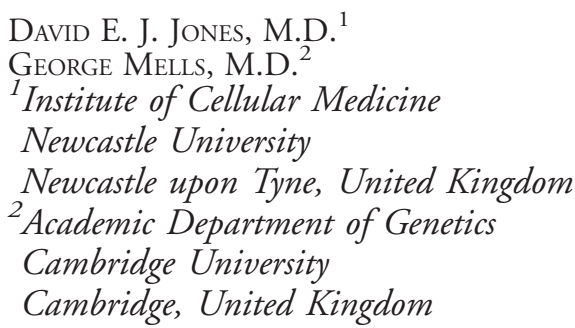

\section{References}

1. Hirschfield GM, Liu X, Xu C, Lu Y, Xie G, Lu Y, et al. Primary biliary cirrhosis associated with HLA, IL12A and IL12RB2 variants. N Engl J Med 2009;360:2544-2555.

2. Hirschfield GM, Liu X, Han Y, Gorlov IP, Lu Y, Xu C, et al. Variant at IRF5-TNPO3, 17q12-21 and MMEL1 are associated with primary biliary cirrhosis. Nat Genet 2010;42:655-657.

3. Liu X, Invernizzi P, Lu Y, Kosoy R, Lu Y, Bianchi I, et al. Genomewide meta-analyses identify three loci associated with primary biliary cirrhosis. Nat Genet 2010;42:658-660.
4. Jones DEJ. Pathogenesis of primary biliary cirrhosis. Gut 2007;56: 1615-1624.

5. Selmi C, Mayo MJ, Bach N, Ishibashi H, Invernizzi P, Gish R, et al. Primary biliary cirrhosis in monozygotic and dizygotic twins: genetics, epigenetics, and environment. Gastroenterology 2004;127:485-492.

6. Jones DE, Watt FE, Metcalf JV, Bassendine MF, James OF. Familial primary biliary cirrhosis reassessed: a geographically-based population study. J Hepatol 1999;30:402-407.

7. Metcalf JV, Bhopal RS, Gray J, James OFW. Incidence and prevalence of primary biliary cirrhosis in the city of Newcastle-upon-Tyne, England. Int J Epidemiol 1997;26:830-836.

8. Goldstein DB. Common genetic variation and human traits. N Engl J Med 2009;360:1696-1698.

9. Nguyen VT, Benveniste EN. Involvement of STAT-1 and Ets family members in interferon-gamma induction of CD40n transcription in microglia/macrophages. J Biol Chem 2000;275:23674-23684.

10. Afford SC, Ahmed-Choudhury J, Randhawa S, Russell C, Youster J, Crosby HA, et al. CD40 activation-induced, Fas-dependent apoptosis and $\mathrm{NKkB} / \mathrm{AP} 1$ signaling in human intrahepatic biliary epithelial cells. FASEB J 2001;15:2345-2354.

11. Nagasawa M, Schmidlin H, Hazekamp MG, Schotte R, Blom B. Development of human plasmacytoid dendritic cells depends on the combined actions of the basic helix-loop-helix factor E2-2 and the Ets factor Spi-B. Eur J Immunol 2008;38:2389-23400.

12. Ouyang X, Negishi H, Takeda R, Fujita Y, Taniguchi T, Honda K. Co-operation between MyD88 and TRIF pathways in TLR synergy via IRF5 activation. Biochem Biophys Res Commun 2007;354:1045-1051.

13. $\mathrm{Hu}$ G, Barnes BJ. IRF-5 is a mediator of the death receptor-induced apoptotic signaling pathway. J Biol Chem 2009;284:2767-2777.

14. Feng D, Stone RC, Eloranta ML, Sangster-Guity N, Nordmark G, Sigurdsson S, et al. Genetic variants and disease-associated factors contribute to enhanced interferon regulatory factor 5 expression in blood cells of patients with systemic lupus erythematosus. Arthritis Rheum 2010;62:562-573

15. Miceli-Richard C, Comets E, Loisseau P, Puechal X, Hachulla E, Mariette X. Association of an IRF5 gene functional polymorphism with Sjögren's syndrome. Arthritis Rheum 2007;56:3989-3994.

16. Watt FE, James OFW, Jones DEJ. Patterns of autoimmunity in PBC patients and their families. QJM 2004;97:397-406.

17. Zhang R, Sun P, Jiang Y, Chen Z, Huang C, Zhang X, et al. Genome-wide haplotype association analysis and gene prioritization identify CCL3 as a risk locus for rheumatoid arthritis. Int J Immunogenet 2010;37:273-278.

18. Yeaman SJ, Kirby JA, Jones DEJ. Autoreactive responses to pyruvate dehydrogenase complex in the pathogenesis of primary biliary cirrhosis. Immunol Rev 2000;174:238-249.

19. Gershwin ME, Mackay IR, Sturgess A, Coppel RL. Identification and specificity of a cDNA encoding $70 \mathrm{kD}$ mitochondrial antigen recognized in primary biliary cirrhosis. J Immunol 1987;138:3525-3531.

20. Yeaman SJ, Fussey SP, Danner DJ, James OFW, Mutimer DJ, Bassendine MF. Primary biliary cirrhosis: identification of two major M2 mitochondrial autoantigens. Lancet 1988;1:1067-1070.

21. Mackay IR. Primary biliary cirrhosis showing a high titre of autoantibody: report of a case study. N Engl J Med 1958;258:185-188.

Copyright $(C) 2011$ by the American Association for the Study of Liver Diseases. View this article online at wileyonlinelibrary.com. DOI 10.1002/hep.24096

Potential conflict of interest: Nothing to report. 